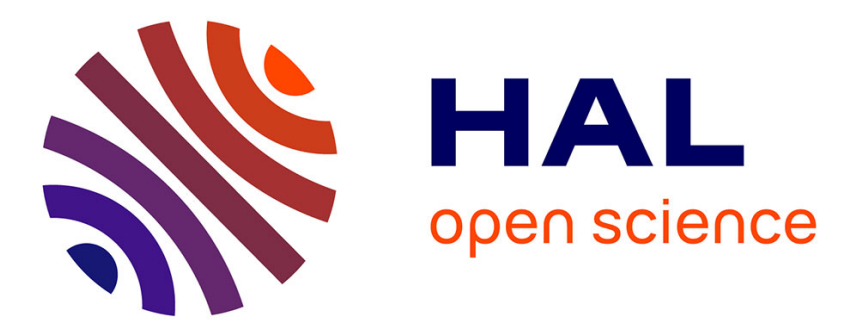

\title{
Investigation on the Magnetic Noise of Stacked Magnetostricitive-Piezoelectric Laminated Composites
}

Xin Zhuang, Sebastien Saez, Marc Lam Chok Sing, Christophe Cordier, Christophe Dolabdjian, Jiefang Li, Dwight Viehland

\section{- To cite this version:}

Xin Zhuang, Sebastien Saez, Marc Lam Chok Sing, Christophe Cordier, Christophe Dolabdjian, et al.. Investigation on the Magnetic Noise of Stacked Magnetostricitive-Piezoelectric Laminated Composites. Sensor letters, 2012, 10, pp.1-5. hal-01061697

\section{HAL Id: hal-01061697 https://hal.science/hal-01061697}

Submitted on 19 Jun 2015

HAL is a multi-disciplinary open access archive for the deposit and dissemination of scientific research documents, whether they are published or not. The documents may come from teaching and research institutions in France or abroad, or from public or private research centers.
L'archive ouverte pluridisciplinaire HAL, est destinée au dépôt et à la diffusion de documents scientifiques de niveau recherche, publiés ou non, émanant des établissements d'enseignement et de recherche français ou étrangers, des laboratoires publics ou privés. 


\title{
Investigation on the magnetic noise of stacked Magnetostricitive- Piezoelectric laminated composites
}

\author{
X. Zhuang ${ }^{1, \text { a }}$, S. Saez ${ }^{1, b}$, M. Lam Chok Sing ${ }^{1, ~ c}$, C. Cordier ${ }^{1}$, \\ d and C. Dolabdjian ${ }^{1, e}$ \\ J. Li ${ }^{2, b}, \mathrm{D}$. Viehland ${ }^{2, \mathrm{c}}$ \\ S. K. Mandal', a , G Sreenivasulu ${ }^{3, b}$ G. Srinivasan ${ }^{3, c}$ \\ ${ }^{1}$ The Groupe de Recherche en Informatique, Image, Automatique et Instrumentation de \\ Caen (GREYC), CNRS UMR 6072 - ENSICAEN and the University of Caen Basse \\ Normandie, France 14050 Caen Cedex \\ ${ }^{2}$ The Materials Science and Engineering, Virginia Tech, Virginia 24061, USA \\ ${ }^{3}$ Physics Department, Oakland University, Rochester, MI 48309 \\ axin.Zhuang@greyc.ensicaen.fr, ${ }^{\mathrm{b}}$ ssaez@greyc.ensicaen.fr, ${ }^{\mathrm{C}}$ marc.lam@greyc.ensicaen.fr, \\ 'christophe.cordier@greyc.ensicaen.fr, ${ }^{\mathrm{e}}$ christophe.dolabdjian@unicaen.fr \\ ajiefang@mse.vt.edu, ${ }^{b}$ viehland@mse.vt.edu \\ asaniitkgp2007@gmail.com, bollapud@oakland.edu, 'srinivas@oakland.edu
}

Keywords: ME sensor; ME charge coefficient; Noise analyses, Charge amplifier.

\begin{abstract}
:
The performance of multi-segments magneto-electric (ME) layered sensors as charge sources has been investigated by using a typical charge amplifier. We found that the intrinsic performance of such sensors consisting of several (namely 3 and 5) segments, is the same whether these segments are connected in parallel or in series. Both, the signal detecting capacity and the intrinsic noise source of ME layered sensors depend on the volume of sensor. We have measured an equivalent magnetic noise of about $1 \mathrm{nT} \sqrt{ } \mathrm{Hz}$ at $1 \mathrm{~Hz}$ and as low as $10 \mathrm{pT} \sqrt{\mathrm{Hz}}$ in the white noise region.
\end{abstract}

\section{Introduction:}

Large magneto-electric (ME) effects can be achieved in layered magnetostrictive and piezoelectric materials [1]. Magnetic signals are transformed into electronic signals via intermediate stress and strain between the magnetostrictive and piezoelectric layers [2], [3], [4] and the generated electric charges can be collected from the polarization surface of the piezoelectric layers. The charge generating capacity is an important factor for a ME sensor, and it is characterized as the ME coefficient $\alpha_{M E}$ [5]. In general, the sensitivity of the ME 
sensor is directly dominated by this coefficient. In order to detect very small signals, some electronics are required to amplify the detected magnetic signals [6], [9].

Noise is another important factor that limits the sensor sensitivity [7]. A lower noise level ensures a better sensor resolution. Noise results from natural random phenomena. Care must be taken in the three following aspects for ME layered sensors. Firstly, the piezoelectric material being a non-perfect insulator, electric charges can move in the piezoelectric layers, thereby producing thermal noise. Such noise exists in piezoelectric layers, although it is negligible. Secondly, due to the relaxation effect between electric displacement and field, a relaxation current noise appears in the piezoelectric layers [5], [10], [11], which is also called dielectric loss noise. The level of this noise source is proportional to the dielectric loss factor of the piezoelectric, equivalent capacitor of sensor. Thirdly, noise sources from the detection electronics must be also considered [6], [9]. A well designed low noise detection circuit will have a noise contribution much lower than that of the sensor.

In the following sections, we present the measured performances of multi-segment $\mathrm{ME}$ layered sensors and discuss the results by using a circuit model. The sensitivities and equivalent magnetic noise of sensors are also analyzed and compared to those given by our model.

\section{Modeling and analysis for ME sensors with associated detecting circuit:}

The ME sensor can be modeled as a charge generator in parallel with the equivalent sensor impedance consisting of a capacitor $C$ in parallel with a resistor $R$ [8]. Because of the insulating properties of the piezoelectric material, the value of the resistor $R$ is quite large $(\sim \mathrm{T} \Omega)$, so that its thermal noise contribution can be considered negligible. The performance of a ME sensor is determined by its magnetoelectric coupling coefficient [3], [11]. In general, two types of ME coefficients are used to represent the ME coupling. One is the ME voltage coefficient which represents the relation between output electric voltage and input magnetic field; the other, called the ME charge coefficient, is used to characterize the charge generation capability of ME sensors. In order to observe the small magnetic signals detected by the ME sensor, a low noise charge amplifier is used in our experiments.

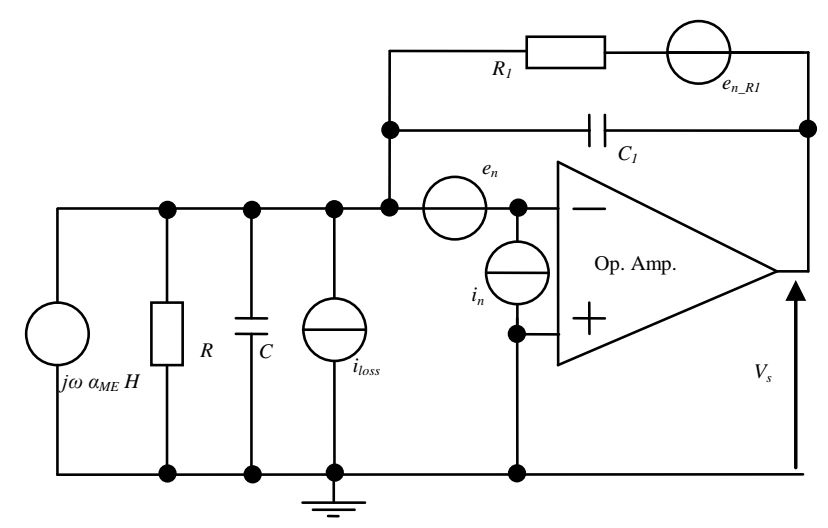

Figure 1: Noise model of the ME sensor and associated Op. Amp. 
The figure 1 shows our experimental set-up involving a ME sensor and a charge amplifier, including the different noise sources. Here, the ME sensor is modeled as a charge generator, in parallel with a capacitor $C$ and a resistor $R . H$ is the applied magnetic field and " $\alpha_{M E}$ " is the charge coefficient. In our experiments, a large feedback resistor $R_{l}(10 \mathrm{G} \Omega)$ was used in parallel with the feedback capacitor $C_{1}(100 \mathrm{pF})$ in order to obtain a sufficiently large output voltage. For the low noise amplifier, we have chosen the LTC6240 from Linear Technology: equivalent input noise voltage $e_{n}=7 \mathrm{nV} / \sqrt{\mathrm{Hz}}$ with a low corner frequency $f_{c}=10 \mathrm{~Hz}$ and equivalent input noise current $i_{n}=0.6 \mathrm{fA} / \sqrt{ } \mathrm{Hz}$. This setup was used to test our ME samples.

The noise level for the ME sensors and amplifier circuit depend mainly on $i$ ) the environmental noise $i$ ) the voltage noise $e_{n}$ and current noise $i_{n}$ of the amplifier and iii) the thermal noise from resistor $R_{l}$ and loss noise from capacitor $C$.

\section{Theoretical analysis:}

The most important factor for a sensor is its transfer function, since a high transfer function can readily amplify small sensed magnetic signals. Thus, the performance can be directly improved by a higher transfer function value.

The transfer function depends on the charge coefficient ${ }_{M E}$ which is given in $(\mathrm{C} / \mathrm{T})$. With the help of the equivalent circuit model [3] [11], the charge coefficient of LT-mode ME sensor can be written as

$$
\alpha_{M E}=\frac{n l w d_{33, m} d_{31, p}}{n s_{11, p}^{E}+(1-n) s_{33, m}^{H}}\left[\mathrm{C} / \mathrm{A} \cdot \mathrm{m}^{-1}\right]
$$

where $n$ is the ratio of the thickness of the magnetic layers to the total thickness, $l$ and $w$ are the length and width of ME sensor, $d_{33, m}, d_{31, p}, s_{11, p}^{E}$ and $s_{33, m}^{H}$ are the magnetostrictive constant, the piezoelectric constant, the flexibility coefficients of piezoelectric and magnetostrictive layers, respectively. We find that the transfer function is proportional to the electric charge generating surface area of the piezoelectric layers as confirmed by the experiments in the next section.

From the Eq. 1, we can write the charge coefficient $\left(\alpha_{M E_{-} \text {para }}\right)$ for a ME layered sensor with $m$ piezoelectric segments in parallel as

$$
\alpha_{M E_{-} \text {para }}=m \frac{n l w d_{33, m} d_{31, p}}{n s_{11, p}^{E}+(1-n) s_{33, m}^{H}}\left[\mathrm{C} / \mathrm{A} \cdot \mathrm{m}^{-1}\right]
$$

which is $m$ times larger than that of a single ME segment sensor. On the other hand, the charge coefficient ( $\alpha_{M E_{-} \text {series }}$ ) for a ME sensor composed of $m$ identical segments connected in series will be the same as that given by each single segment ME sensor as can be deduced from Eq. 1. 
The ratio of the charge coefficients for the parallel connection and series connection is:

$$
\frac{T_{r_{-} \text {para }}}{T_{r_{-} \text {series }}}=\frac{\alpha_{M E_{-} \text {para }}}{\alpha_{M E_{-} \text {series }}}=m
$$

showing that the charge coefficient for $m$ parallel segments sensor is $m$ times larger than that of a $m$-segments series connected sensor.

If we consider the equivalent sensor capacity of above configuration, the capacity for the parallel assembly is given as $C_{\text {para }}=m \frac{l w \varepsilon_{33}^{S}}{t_{p}}$, whereas in the case of $m$ segments in series, we have $C_{\text {series }}=\frac{l w \varepsilon_{33}^{S}}{m t_{p}}$. Thus, the equivalent sensor capacity for parallel connection is $m^{2}$ times larger than that given by the series connection as

$$
\frac{C_{\text {para }}}{C_{\text {series }}}=m^{2}
$$

The intrinsic sensor noise mainly comes from the dielectric loss current in the piezoelectric layers [5], [10], [11], the sensor noise contribution to the output voltage in figue 1 can be written as $e_{n_{-} T}^{2}(f)=\left|Z_{1}\right|^{2} i_{n_{-} \text {loss }}^{2}(f)$, where $i_{n_{-} \text {loss }}(f)=\sqrt{4 k_{B} T \omega C \operatorname{Tan} \delta(f)}$. Here, $k_{B}$ is the Boltzmann constant, $T$ is the temperature in Kelvin, $\omega$ is angular frequency, $\tan (\delta(f))$ is the dielectric loss factor of the piezoelectric layers, and $Z_{1}=\frac{R_{1}}{1+j(2 \pi f) C_{1} R_{1}}$ is the transfer function for the dielectric loss noise source of the sensor.

The ratio of the noise given a $m$ parallel segment sensor to that of the series configuration is

$$
\frac{e_{n_{-} \text {loss_para }}(f)}{e_{n_{-} l o s s_{\_} \text {series }}(f)}=\frac{i_{n_{-} \text {loss_para }}(f)}{i_{n_{-} \text {loss_series }}(f)}=m .
$$

In the white noise region, the output voltage noise of the op-amp gives the main noise contribution, which is described by the following formula [9]

$$
e_{n}(f)=e_{n_{-} a m p}(f) \frac{C_{1}+C}{C_{1}} .
$$

The ratio between the output voltage white noise levels for the parallel and series modes is given as

$$
\frac{e_{n_{-} \text {white_para }}(f)}{e_{n_{-} \text {white_series }}(f)}=\frac{C_{1}+C_{\text {para }}}{C_{1}+C_{\text {series }}} .
$$


The performances of ME sensors are evaluated by their equivalent input magnetic noise when the latter is predominant. The noise ratio between $m$-segments parallel connection and series connection is shown as follows

$$
\frac{b_{n_{-} l o s s_{\text {ppara }}}(f)}{b_{n_{-} \text {loss_series }}(f)}=\frac{i_{n_{-} \text {loss_para }}(f) / \alpha_{M E_{-} \text {para }}^{Q}}{i_{n_{-} \text {loss_series }}(f) / \alpha_{M E_{-} \text {series }}^{Q}}=1 .
$$

This formula tells us that the performance of $m$-segment for a parallel or a series connection is identical when the intrinsic sensor noise is the dominant noise source.

For a non-perfect designed circuit, the equivalent voltage noise $e_{n_{-} a m p}(f)$ of the amplifier is the dominant noise source. Thus, the equivalent input magnetic noise ratio of $m$ segments in parallel and series connection is

$$
\frac{b_{n_{-} \text {para }}(f)}{b_{n_{-} \text {series }}(f)}=\frac{e_{n_{-} \text {para }}(f) / \alpha_{M E_{-} \text {para }}^{Q}}{e_{n_{-} \text {series }}(f) / \alpha_{M E_{-} \text {series }}^{Q}}=\frac{1}{m} \frac{C_{1}+C_{\text {para }}}{C_{1}+C_{\text {series }}} .
$$

Under these conditions, the larger the capacitor the lower is the performance [9]. In the case where $C_{\text {para }}>>C_{1}$ and $C_{\text {series }}>>C_{1}$, we have

$$
\frac{b_{n_{-} \text {para }}(f)}{b_{n_{-} \text {series }}(f)} \approx m \text {. }
$$

\section{Samples:}

The samples, we used, are trilayers of ferromagnetic alloy-PZT-ferromagnetic alloy. The alloy is $49 \% \mathrm{Fe}, 49 \% \mathrm{Co}, 2 \% \mathrm{~V}$ with high magnetostriction alloy called Permendur. The thickness is $0.016 \mathrm{~cm}$. The PZT of dimensions $5 \mathrm{~cm} \times 1 \mathrm{~cm} \times 0.04 \mathrm{~cm}$ is a commercial product (\#851 from American Piezoceramics). The fabrication of the ME composites contained the following steps: i) Formed Permendur and PZT layers are cleaned by the acetone solution in order to achieve a good connection between layers. ii) Epoxy glue is spread on the surfaces of magnetostrictive and piezoelectric layers, the two phases of materials are stacked together and fixed with clips. iii) Laminated composites are sintered under a certain temperature for several hours. After the fabrication procedure, electrodes are connected onto composites.

\section{Experimental results and discussions:}

During our measurements, the sensors were placed at the centre of a pair of Helmholtz coils, along the sensing direction of sensor as shown in Fig.2. A pair of permanent magnets attached near the two ends of the sensor was used to apply a biasing magnetic field along the sensing sensor axis. The magnetic field generated by the Helmholtz coils was obtained by means of a function generator and a resistor $R_{a}(1 \mathrm{k} \Omega)$. A spectrum analyzer (HP 3562A) and an oscilloscope were used to measure the output signals. The applied magnetic field was 
obtained by measuring $V_{b}$, the voltage across $R_{b}$. In order to reduce the noise level for our experiments, the Helmholtz coils, sensor, charge amplifier and another optional preamplifier (EG\&G 5113 PRE-AMP) were placed inside our shielded room.

\section{A Transfer functions by using charge circuit}

In this section, the experimental results of the transfer function for a parallel and a series connection of several ME segments will be compared and discussed. Two LT-mode layered ME sensors, presenting a mechanical resonance around $40 \mathrm{kHz}$, were tested by using the measurement setup described in the preceding paragraph. "Sensor 1" consists of three segments whereas "sensor 2" has five segments. Fig. 2 and Fig. 3 show the measured transfer functions of "Sensor 1" and "Sensor 2", respectively. The transfer functions of the parallel connection sensors are $m$ times higher than transfer functions of the series connection sensors, since the one in parallel connection have $m(=3$ or 5$)$ times larger surface than in series connection.

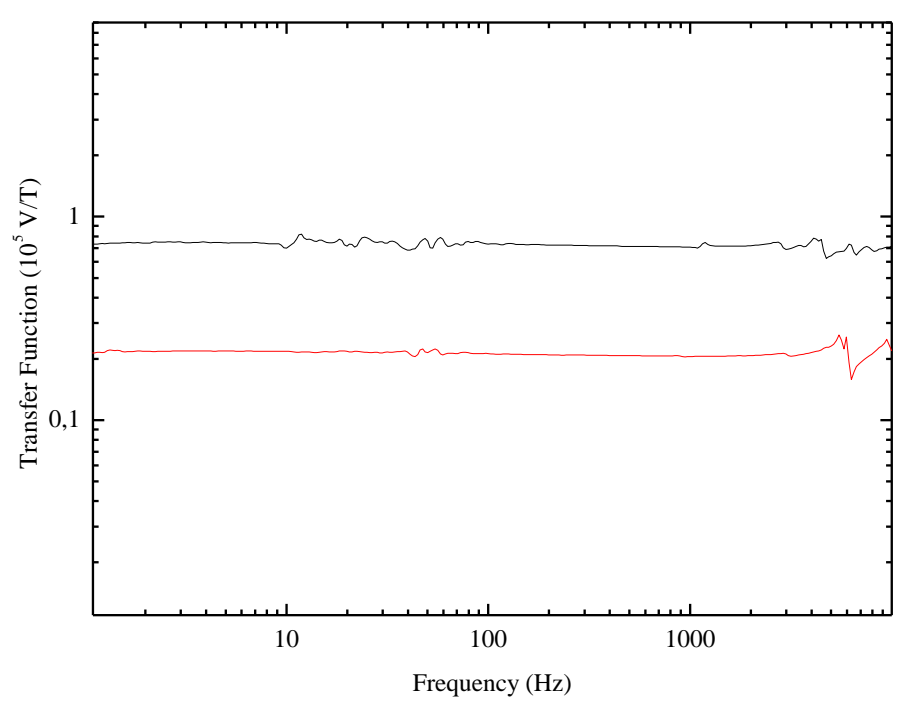

Figure2: Transfer function of sensor 1 (3 segments) for a parallel connection (black curve), and a series connection (red curve) as a function of frequency by using amplifier LTC6240 Op. Amp. with $R_{1}=10 \mathrm{G} \Omega, C_{1}=100 \mathrm{pF}$. The level of the black curve is nearly 3 times higher than the red one. This is excepted with Eq. 2, charge coefficient is proportional to the surface of the sensor. 


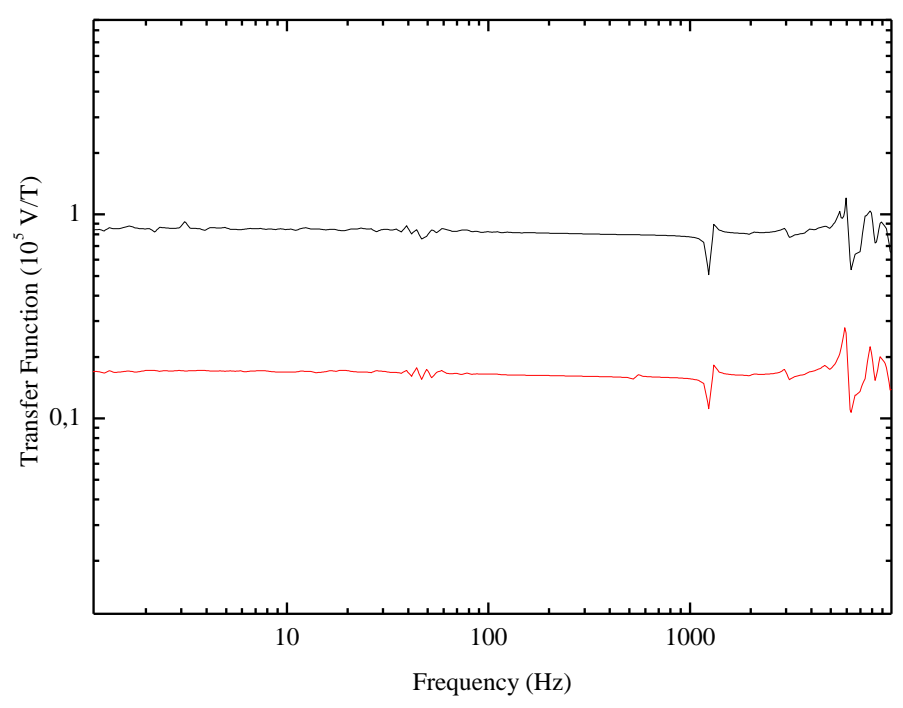

Figure 3: Transfer function of sensor 2 (5 segments) for a parallel connection (black curve), and a series connection (red curve) as a function of frequency by using the same measurement configuration in Fig. 2.

The black curves, both in Fig. 2 and Fig. 3, are about $m(=3$ or 5$)$ times higher than the red ones, as expected from Eq. 5.

\section{B Equivalent input magnetic noise}

Equivalent input magnetic noise defines the performance of a magnetic sensor. A lower equivalent input magnetic noise gives a better sensor performance. The input equivalent magnetic noise for $m(=3$ or 5$)$ segments in parallel connection have the same level in low frequency, because it depends only on the sensor volume. Meanwhile, in the white noise region, a ratio of $\frac{b_{n_{-} \text {para }}(f)}{b_{n_{-} \text {series }}(f)}=\frac{1}{m} \frac{C_{1}+C_{\text {para }}}{C_{1}+C_{\text {series }}} \approx m$, if $C_{1}<<C$.

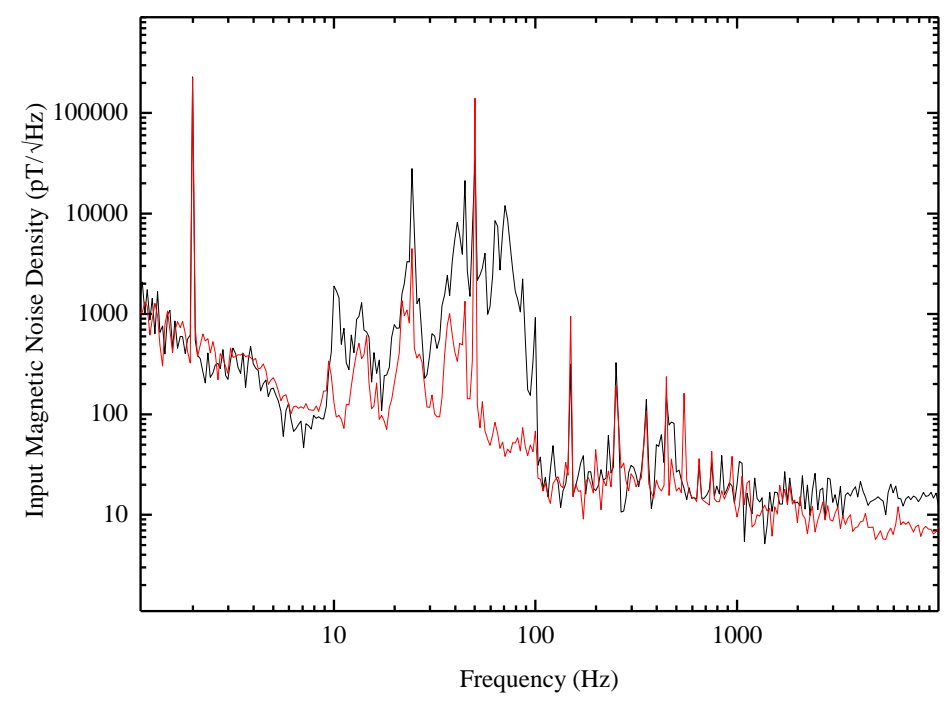


Figure4: Equivalent input magnetic noise density of sensor 1 (3 segments) for the parallel connection (black curve) and the series connection (red curve) as a function of frequency. The measurement configuration is unchanged.

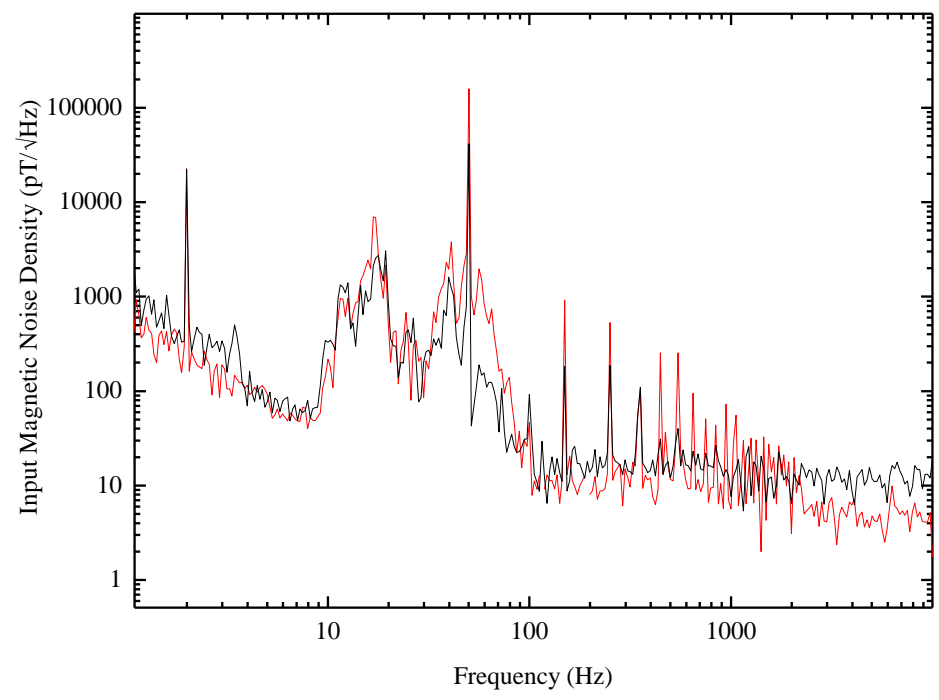

Figure5: Equivalent input magnetic noise density of sensor 2 (5 segments) in parallel connection (black curve) and series connection (red curve) as a function of frequency by using an LTC6240 Op. Amp. with $R_{1}=10 \mathrm{G} \Omega, C_{l}=100 \mathrm{pF}$.

The same equivalent input magnetic noise level is found in figures 4 and 5 at low frequency $(<1 \mathrm{kHz}$ ), meanwhile, at white bandwidth (around $10 \mathrm{kHz}$ ) curves show a factor of 3 and 5 . This corresponds to the theoretical prediction we expected. The synthesis of measured data is given in Table 1 and Table 2. 
Table 1: Experimental and theoretical parameters of sensors in parallel and series connection modes.

\begin{tabular}{|c|c|c|c|c|c|c|}
\hline & \multirow{2}{*}{$\begin{array}{c}C_{\text {para }} \text { or } C_{\text {series }} \\
(\mathrm{nF})\end{array}$} & \multicolumn{2}{|c|}{$\frac{C_{\text {para }}}{C_{\text {series }}}$} & \multirow{2}{*}{$\begin{array}{c}\text { Charge } \\
\text { coefficient } \\
\alpha_{\mathrm{ME}} \\
(\mu \mathrm{C} / \mathrm{T})\end{array}$} & \multicolumn{2}{|c|}{$\frac{\alpha_{M E_{-} \text {para }}}{\alpha_{M E_{-} \text {series }}}$} \\
\hline & & experimental & theoretical & & experimental & theoretical \\
\hline $\begin{array}{l}3 \text { segments } \\
\text { in parallel }\end{array}$ & 17.2 & \multirow{2}{*}{8.6} & \multirow{2}{*}{9} & 7.1 & \multirow{2}{*}{3.23} & \multirow{2}{*}{3} \\
\hline $\begin{array}{l}3 \text { segments } \\
\text { in series }\end{array}$ & 2 & & & 2.2 & & \\
\hline $\begin{array}{l}5 \text { segments } \\
\text { in parallel }\end{array}$ & 17.2 & \multirow{2}{*}{23} & \multirow{2}{*}{25} & 8.5 & \multirow{2}{*}{5} & \multirow{2}{*}{5} \\
\hline $\begin{array}{l}5 \text { segments } \\
\text { in series }\end{array}$ & 0.75 & & & 1.7 & & \\
\hline
\end{tabular}

Table 2: Experimental and theoretical equivalent magnetic noise levels in parallel and series connection modes.

\begin{tabular}{|c|c|c|c|c|c|c|}
\hline & \multirow{2}{*}{$\begin{array}{c}\text { Equivalent } \\
\text { magnetic } \\
\text { noise at } 1 \mathrm{~Hz} \\
b_{n \_l}{ }^{2} \\
(\mathrm{nT} / \sqrt{\mathrm{Hz}})\end{array}$} & \multicolumn{2}{|c|}{$\frac{b_{n_{-} 1 H z_{-} \text {para }}}{b_{n_{-} 1 H z_{-} \text {series }}}$} & \multirow{2}{*}{$\begin{array}{c}\text { Equivalent } \\
\text { magnetic } \\
\text { noise at } \\
\text { white } \\
\text { bandwidth } \\
b_{n_{-} w h i t e} \\
(\mathrm{pT} / \sqrt{\mathrm{Hz}})\end{array}$} & \multicolumn{2}{|c|}{$\frac{b_{n_{-} \text {white_para }}}{b_{n_{-} \text {white_series }}}$} \\
\hline & & experimental & theoretical & & experimental & theoretical \\
\hline $\begin{array}{l}\text { 3segments } \\
\text { in parallel }\end{array}$ & 1.1 & \multirow{2}{*}{1} & \multirow{2}{*}{1} & 16.5 & \multirow{2}{*}{2.29} & \multirow{2}{*}{2.75} \\
\hline $\begin{array}{c}3 \text { segments } \\
\text { in series }\end{array}$ & 1.1 & & & 7 & & \\
\hline $\begin{array}{l}5 \text { segments } \\
\text { in parallel }\end{array}$ & 0.9 & \multirow{2}{*}{1.13} & \multirow{2}{*}{1} & 14 & \multirow{2}{*}{4} & \multirow{2}{*}{4.071} \\
\hline $\begin{array}{c}5 \text { segments } \\
\text { in series }\end{array}$ & 0.8 & & & 3.5 & & \\
\hline
\end{tabular}

\section{Conclusion:}

Performances of the parallel and series connections of ME segment sensors have been investigated by using a typical charge circuit. The signal transfer function is proportional to the surface where the electric charges are generated. In white noise bandwidth, the output voltage noise is mainly dominated by the equivalent voltage noise source of the charge amplifier. However, dielectric loss noise from piezoelectric layer gives the main noise contribution at low frequency. In term of input magnetic noise, a same level is found at low frequencies. If the capacitor in the amplifying circuit is much smaller than the one of sensor, the white performances are proportional to the segment number of sensor. So, we can conclude that it is better to used ME layered sensors in $m$-segment series connection when the 
white noise level of the charge circuit is non-negligible. Notice that the intrinsic noise of the sensor is unchanged in $m$-segment series or parallel connections.

This work was supported by the U.S. Defense Advanced Research Projects Agency (DARPA).

\section{References:}

[1] C. Nan, Phys. Rev. B, 50, 6082-6088 (1994).

[2] C. Nan, M. I. Bichurin, S. Dong, D. Viehland, G. Srinivasan, J. Appl. Phys. 103, 031101 (2008).

[3] S. X. Dong, J. F. Li, and D. Viehland, IEEE Trans. Ultrason. Ferroeletr. Freq. Control 50, 1253 (2003).

[4] Pyu J., Priya S., Carazo A. Vazquez, Uchino K., Kim H. E., Magnetoelectric Properties in Piezoelectric and Magnetostrictive Laminate Composites Jpn. J. Appl. Phys. Vol. 40 (2001) pp.4948-4951

[5] Z. Xing, J. Li, and D. Viehland, Modeling and the signal-to-noise ratio research of magnetoelectric sensorsat low frequency, Appl. Phy. Lett. 91, 142905 (2007)

[6] Z. P. Xing, J. Y. Zhai, S. X. Dong, J. F. Li, D. Viehland, and W. G. Odendaal, Modeling and detection of quasi-static nanotesla magnetic field variations using magnetoelectric laminate sensors, Meas. Sci. Technol. 19, 015206 (2008)

[7] C. D. Motchenbacher and J. A. Connelly, Low noise Electronic System Design (Wiley, New York, 1993), Chap 1.

[8] J. Zhai, Z. Xing, S. Dong, J. Li, and D. Viehland, J. Am. Ceram. Soc., 91 [2] 351-358 (2008).

[9] X. Zhuang, M. Lam Chok Sing, C. Cordier, S. Saez, and C. Dolabdjian; J. Das, J. Gao, J. F. Li and D. Viehland, Characterization of Metglas/PZT fiber laminate composites as a magnetic sensor, to be published in IEEE Sensors, 2011

[10] X. Zhuang, M. Lam Chok Sing, C. Cordier, S. Saez, and C. Dolabdjian; L. Shen, J. F. Li, M. Li, and D. Viehland, Efficiency of applied axial field modulation technique on ME sensor input magnetic noise, to be published in IEEE Sensors, 2011

[11] X. Zhuang, M. Lam Chok Sing, C. Cordier, S. Saez, and C. Dolabdjian; L. Shen, J. F. Li, M. Li, and D. Viehland, Theoretical performance modeling for ME laminate composites with charge and voltage detection electronics, to be published in J. Appl. Phy., 2011 